quiet. To which might be added the lamentable fact that none of the twentyfive collisions which have occurred in the Dover Strait between June 1967 and November 1969 has produced any lessons. Nothing is known about the circumstances, the legal decisions, and the tribunals. The degree to which the attribution of responsibility takes into account the recommendations to follow a particular route is totally ignored. Is it, one might ask, by this silence that shipowners and insurance interests hope to profit from this. water-shed in the history of navigation?

Since 1967 the idea of recommended routes has spread like wildfire. Having been engendered in an atmosphere of almost total indifference, it is now presented as a panacea for the prevention, without effort, of the risk of collision. This phenomenon is not new; it is exactly what happened in the case of radar twenty years ago. No more than radar, should traffic separation be an invitation to rest on the laurels of an ephemeral success. What we know today is simply a rough plan. It has yielded the results that were expected of it as a matter of urgency. But from the very beginning one could foretell difficulties which would arise as a matter of second urgency. At the time, one said let us achieve the essential and bother about the minor difficulties later. Now it is later, and the foretold difficulties have arisen, with others that were not foretold. It is time to stop diversionary activities and to pursue the work undertaken with tenacity and in the spirit in which it was undertaken. This is the only way in which to perfect the plan.

\title{
REFERENCES
}

1 Richey, M. W. (1970). Reversing the Dover Strait routes (Review). This Journal, 23, 272.

2 Oudet, L. (1970). Accident black spots in the Lower North Sea. This Journal, 23, 108.

\section{An Examination of some Ship Radars with Automatic Computation}

\author{
Captain F. J. Wylie, R.N. (retd)
}

IT is generally accepted nowadays that, to obtain full value for anti-collision purposes from the use of radar by a ship proceeding in poor visibility, it is necessary to plot, or to compute in some other way, the raw data which may be obtained from the radar. It is also usually conceded that, for this to be done effectively in frequented areas, without the aid of mechano/electronic devices, a great deal of time and expertise is demanded from the radar observer and a great deal of responsibility thereby rests upon his shoulders.

To reduce the load of work and the possibility of human error and to give the observer more time to use his intelligence in appraising the situation and keeping it under review, radar engineers are already pressing forward the development of automatic devices which, in one way or another, produce computed information. in the form ultimately needed. 
The devices which come to mind at the present time are:

(i) Marconi Predictor; 1, 2, 3

(ii) G.E.C.-A.E.I. Compact; 2

(iii) Kelvin Hughes H.R.D. 2, 4 (abbreviation used in this article for History Recording Display):

(iv) Cerci A.T.A.W. ; ${ }^{5}$

(v) Iotron Digiplot.

Some of these have done sea trials, some are doing them and others have not yet reached that stage. But, since in many ways they differ widely, it may be interesting at this early stage to see what they will or will not do. What seemed to be the basic requirements were summarized very briefly in an article by the present writer in this Journal in $1965 ;^{6}$ this will be used later. Meanwhile, it may be as well to describe each of the five examples but first a point of nomenclature needs to be made clear. The word 'track' is used by this writer to denote a path astern of the echo which indicates the movements, either true or relative, on the PPI over the past few minutes. Many engineers use the term 'tracking radar' to denote one in which an echo, once 'gated', is followed by the electronic gate, however it may move; the data thus gained may, when computed, be shown on the PPI in the form of velocity vectors, indicating the latest computed course and speed only and not past history. In this note this kind of radar is called a 'vectoring 'equipment.

The Marconi Predictor is a complete radar set, which records all echoes on a magnetic tape and replays it so as to repaint a number of the past positions of each echo and provide a track of past movement. Three past positions, separated by $\frac{1}{2} \mathrm{~min}$., I min. or $2 \mathrm{~min}$. intervals, are shown in addition to the current position of the echo. One of these four positions is painted on every sweep, so that the whole track is up-dated every 10 secs. The tracks may be true or relative or predicted relative, the last being based upon a proposed alteration of course and/or speed.

The G.E.C.-A.E.I. Compact is a 'lock-on and follow' system which operates in association with an Escort radar and will deal with up to twelve echoes selected and gated by the operator, who places an illuminated ring round each selected echo. The computer receives the successive coordinates of the echoes' positions and displays the computed information in the form of velocity vectors (called 'track ahead' by the makers), whose direction is the course and whose length represents the distance run in a selected time, i.e. the speed of the echo movement. The vectors may be true or relative at choice. In true motion, own ship is given an appropriate vector. They are displayed continuously in green on a separate cathode ray tube, superimposed by reflection over the orange echo paints on the Escort PPI. The operator may select a C.P.A. distance to suit the circumstances and have this shown as a circle round own ship's position, i.e. round the centre of the PPI in relative motion and at the end of own ship's velocity vector in true motion.

The Kelvin Hughes H.R.D. operates in association with a K.H. photoplot system (PRP) to which a special cathode ray tube and image-retaining panel are added, and these store a photographic picture of past movement which is superimposed on the current picture and so projected with it on to the plotting surface. The system is such that the past track is built up over a period of 4,8 or 20 minutes 
and then erased and recommenced. The track may be true or relative according to choice.

A 'predictor overlay' of transparent perspex is fitted above the projected display and this facilitates manual drawing of relative tracks when the display is in true motion and vice versa, also the predicted change in relative tracks when course is to be altered.

The Cerci A.T.A.W. is a 'lock-on and follow' system, operating on the video output of a Raytheon radar and giving its information on the PPI. A video processor determines the exact coordinates of up to seven echoes, selected by the operator, and passes them to the calculator. From the successive positions of each gated echo, identified in this case by a horizontal line, a velocity vector is calculated and shown on the C.R.T. in either the true or the relative mode. Time markers at selected intervals appear on the vectors. The system has three types of display:

(i) Automatique, in which only selected (gated) echoes, which will pass within a certain pre-selected C.P.A. distance, are shown.

(ii) Sequentielle, in which each gated echo in turn may be examined and automatic digital indication of the C.P.A. distance and time or the true course and speed given.

(iii) Globale (comprehensive), in which the velocity vectors of all gated echoes are shown, whether dangerous or not.

The lotron Corporation (U.S.A.) Digiplot is a vectoring system which operates on the video output of any conventional radar and displays on a separate C.R.T. It has two digital processors, the first of which discriminates between large (e.g. land) echoes and small (e.g. ship) echoes, passing the coordinates of the former direct to the C.R.T. and those of the latter to the second processor, which estimates the courses and speeds of targets. Current data on each target, i.e. every small echo, at present up to a maximum of 56 , is stored in the magnetic core memory of this processor and the C.P.A. distance and time is computed each time new data is received. This processor also controls the alarm, adjusts the data to suit the scale, the vector length and type of plot, and drives the display. The writing beam of the display repeats the trace fifty times per second, making the picture continuous and flicker free.

The general method of operation of this system is to arrange all the (small) echoes in a sequence depending on the degree of threat of each and to display the most threatening $4^{\circ}$ of them. The degree of threat is based on a C.P.A. distance and time chosen by the operator as the criterion. The information is displayed in the form of a velocity vector, the length of which represents the distance to the future position at the time selected for prediction. The vector may be true or relative; own ship is always at the centre of the C.R.T.; the display may be head up or north up. Enhanced brightness is used on the C.R.T. to distinguish threatening targets from other moving targets. The near edge of land masses are outlined by dots.

A digital indicator is provided to give the range, bearing, course, speed and C.P.A. distance and time for any target whose echo is selected by a light sensitive pointer.

To make any coherent comparison between these sophisticated equipments, it seems necessary to establish some points of principle against which to view 
them. Those suggested in the article ${ }^{6}$ referred to above may serve to rationalize the possibilities, whether or not they themselves are agreed:

'(i) To show on the PPI, on demand and with minimal delay, the tracks of all vessels simultaneously, whose echoes are on the screen.'

The opening words were intended to require the additional intelligence to be shown on the PPI in normal use by the observer/captain, for the reasons given in the article. Predictor and H.R.D. show tracks on the PPI; Compact shows its velocity vectors superimposed on the PPI by reflection; A.T.A.W. displays its information on the radar PPI; Digiplot displays its vectors on a separate C.R.T.

The point at once arises, whether there is any preference between the track and the velocity vector as a means of expressing the computed intelligence. The vector shows no past history, though this might be useful if an alteration of course has recently occurred; the track shows no forward estimate of echo or own ship's position; the circuitry required for vectoring permits further information to be displayed, such as C.P.A. circles. Of course, the more complete the prediction and display of future positions, the greater care will be required to avoid clothing them with certainty.

The requirement for the tracks/vectors to be 'on' or 'off' on demand is satisfied. With Digiplot, the PPI of the parent radar would presumably be available if the vector display were not required.

The degree of satisfaction of the requirement for minimal delay in obtaining tracks and in switching between true and relative varies somewhat. Predictor's delay is ten seconds, assuming the magnetc tape is already running and that a change of scale or track length is not made simultaneously; after such changes the delay depends on the (new) tracklength. In the H.R.D., the tracks are built up sweep by sweep when demanded and the full track will take 4,8 or 20 minutes to build up. Of course, sufficient track may be available long before these times. With the vectoring equipments, the delay depends upon how long the computer is given to obtain a reasonably stable answer from the data. Compact takes $3^{6}$ seconds and A.T.A.W. about 60 seconds. Digiplot is instantaneous.

Finally there is the requirement for 'all vessels simultaneously', which is intended to avoid, for the operator, the burden and anxiety of selecting the most threatening echoes and keeping his selection up-dated. Both the trackers do this, of course. Digiplot does it to the extent that the processors continually review the degree of threat of all echoes and select the chosen number of the most threatening for display. As up to 40 may be demanded, it may be thought that this relieves the operator of the burden. Neither Compact (up to 12 echoes) nor A.T.A.W. (up to $j$ echoes) can be said to meet this requirement. However, provided that circumstances permit one or two spare channels to be available for the purpose, they will provide for the rapid checking of suspect echoes without involving the operator in calculation.

(ii) 'The tracks to be true or relative at choice and to extend over a maximum period to be determined, up to the latest positions of the echoes.' Of course, there is an implication of minimal delay here as in (i).

The rapid change of mode is given in all the equipments except the H.R.D., in which the PPI has to be switched to true motion and the track given time to build up. There are manual facilities in H.R.D. for assisting the drawing of relative tracks when the set is in true motion and vice versa, but this is hardly 
relevant here. The delay in Predictor is 10 seconds for the complete track. With the velocity vector sets the change is virtually instantaneous. All the equipments have either a selected track length or a vector length depending on a selected time and all terminate or originate at the current position of the echo. (In H.R.D. minus 20 seconds.)

(iii) 'The execution of this choice (see (ii)) must not cause any displacement in the positions of the echoes on the screen.' This is to avoid any risk of losing echo identity. It implies that the display must be permanently centred or off-centred. This confers one of the advantages of the automatic tracking or vectoring displays, which do not depend on echo trails for depicting true motion, because the complications of traversing the trace origin and re-setting are avoided as are the operational disadvantages of loss of view ahead and at the side.

The requirement is met in Predictor and Digiplot and it can be met in Compact by setting own speed at zero and centring the display. The same may apply to A.T.A.W. (The natural echo trails will, of course, show relative motion.) In H.R.D., the tracks being projected photographs of the actual display, the normal characteristics of true and relative motion will be reproduced.

(iv) 'The tracks to show time intervals from which speeds may be assessed; an indication of own ship's speed in a similar manner would be a great convenience.'

Predictor and A.T.A.W. show the time intervals selected. In Compact, A.T.A.W. and Digiplot the vector length is the distance run in the total time selected. In H.R.D. the time-lapse dial shows the length in time of the tracks so far built up. All this applies also to own ship's track or velocity vector in true motion.

(v) 'There must be no significant obstruction of the echoes by the tracks.'

Digiplot does not display its information on the main PPI and so is not concerned; Compact's vectors are distinguished by colour from the echo picture and are continuously and finely painted without afterglow; A.T.A.W.'s vectors should not obscure echoes either; Predictor's tracks being a series of dots at regular intervals are unlikely to obstruct the view other than momentarily. The nature of H.R.D.'s tracks lends itself rather more to possible obstruction, particularly on the longer track lengths. On the short track with its erasure every four minutes there should be no problem.

(vi) 'Means should be provided for showing the effect upon the relative tracks of an intended alteration of course and/or speed.'

Predictor, Compact, A.T.A.W. and Digiplot are capable of doing this automatically. Each of them allows in some way for the characteristics of own ship's manœuvring. With Predictor, the prediction may be continued during the turn. Digiplot provides for the display of the complete theoretical execution of the proposed manœuvre, the process being speeded up 30 times. Compact, when in true motion, provides for own ship a dotted vector and C.P.A. circle to indicate a proposed new course and/or speed, which may be seen in conjunction with the unaltered true motion vectors of the gated echoes. With H.R.D., the effect of an alteration has to be predicted manually by using the Predictor overlay.

The article, to which the clauses considered above belong, did not include any requirement for alarms or warnings. The importance of such indications in fully automatic systems is such that an examination of the provision made for them in the equipments mentioned should be of interest:

$\left.\begin{array}{l}\text { Predictor } \\ \text { H.R.D. }\end{array}\right\}$ No provision 
Compact (a) Warning by sound and flashing bearing indicator when any closing echo crosses guard rings at $\mathrm{I}$ I and 9 miles;

(b) Warning by lamp, sound and flashing identification ring when a gated echo will pass inside a pre-selected C.P.A. distance within the next 30 minutes.

(c) A 'Target Lost' lamp informs the operator that tracking a gated target is no longer possible.

A.T.A.W. (a) Warning, sound and visual when, in the absence of the operator from the PPI, an echo closes within a pre-selected distance;

(b) Warning, sound and visual, when the C.P.A. distance of a gated echo will be less than a pre-selected distance;

(c) Warning, sound and visual, if auto-tracking of a gated echo is lost;

(d) Similar warning if the speed of a gated echo is greater than the maximum of which the system is capable.

Digiplot Warning, sound and visual, when any target will pass within a set C.P.A. distance within a set time interval.

Another feature which is not available in all the equipments is the digital readout of computed information in A.T.A.W. and Digiplot.

All the facilities mentioned are summarized in the following table:

TABLE I. SúmMary of Facilities

\begin{tabular}{|c|c|c|c|c|c|}
\hline & I & 2 & 3 & 4 & 5 \\
\hline Track $(T)$ or Vector $(V)$ & $\boldsymbol{V}$ & $\boldsymbol{V}$ & $V$ & $T$ & $T$ \\
\hline Displayed on PPI or separate C.R.T. & C.R.T. & PPI & PPI & PPI & PPI \\
\hline Delay in showing $T$ or $V$ on demand & Nil & $60^{s}$ & $36^{s}$ & $4^{\mathrm{m} *}$ & $10^{5}$ \\
\hline $\begin{aligned} \text { Not required (NR) } \\
\text { Echo selection: } \\
\text { By observer (OB No.) } \\
\text { By computer (CP No.) }\end{aligned}$ & $\mathrm{CP}_{40}$ & $\mathrm{OB}_{7}$ & $\mathrm{OB}_{12}$ & NR & NR \\
\hline Time to change mode, true or relative & Nil & Nil & Nil & $4^{\mathrm{m} *}$ & 10 \\
\hline Display centred in true and relative & Yes & No & May & No & Yes \\
\hline Obscuration of echoes & No & No & No & $\mathrm{S}$ & $\mathrm{L}$ \\
\hline Trial course facilities: automatic & Yes & Yes & Yes & No & Yes \\
\hline Warning of distant echo (audio \& visual) & No & Yes & Yes & No & No \\
\hline Warning of collision risk (audio \& visual) & Yes & Yes & Yes & No & No \\
\hline Digital read-out of computed inform & Yes & Yes & No & No & No \\
\hline
\end{tabular}

$1=$ Digiplot $4=$ H.R.D.

$2=$ A.T.A.W. $5=$ Predictor

$3=$ Compact
*Correspondingly longer on longer track lengths $\mathrm{S}=$ Some $\mathrm{L}=$ Little 
REFEREN CES

1 Wylie, F. J. (1968). The Use of Radar at Sea, p. 243.

2 Wylie, F. J. (1970). Choosing and Using Ship's Radar, Ch. I.

3 Watt, J. and Piercy, B. C. (1969). A new marine radar display system. This Journal, 22, 205 .

4 Embling, C. A. and Stewart, J. P. (1968). A history recording radar display with prediction. This Journal, 21, 471 .

5 Les moyens automatiques: l'anti-collision ATAW, Navigation (Paris), 17, 311 , July 1969 .

6 Wylie, F. J. (1965). Radar as an anti-collision aid: the ultimate essentials in presentation. This Journal, 18, 232 .

\title{
Spherical Triangles with Integral-degree Parts
}

\author{
D. H. Sadler
}

IN certain esoteric mathematical and computing circles there is, or used to be before the advent of the electronic computer, an interest in near-integral solutions of incommensurable problems. Apart from aesthetic considerations such solutions can have valuable applications in the checking of methods of computation; this is the excuse for the following note.

The investigation from which it arises was itself a by-product. As part of the British contribution to the new Sight Reduction Tables for Marine Navigation (H.O. 229 in U.S.A.; H.D. 605 in the United Kingdom) it was necessary to examine carefully each of the approximately 20 million figures in the six volumes; although the setting was computer-controlled and numerically checked, only visual examination could detect indistinct or broken figures. Such examination is extremely arduous and calls for great powers of concentration if its purpose is not to be wasted. To provide an incentive prizes were offered for finding the combinations of altitude and azimuth that were closest to integral degrees; the examiners could, of course, only pick out the combinations of altitudes ending in 00.0 and azimuths ending in $0 \%$ (about $I$ in 6000), and the closest is then a matter of chance, determined by recomputationproviding incidentally a series of independent checks.

An analysis of the 20-30 closest pairs (with tolerances of about \pm 0.0005 ; many are not independent) immediately reveals what would have been obvious in a direct investigation, namely that they all belong to two special classes; these are described below as $(a)$ and $(b)$.

Class $(a)$ is the more interesting since all six parts of the spherical triangle are near-integral; two angles are equal respectively to their opposite sides, while the sum of the third angle and its opposite side is $180^{\circ}$. The three best examples are tabulated in Table 1 . 